\section{Bone loss, pain and inflammation: three faces of ACPA in RA pathogenesis}

\author{
Jeremy Sokolove, ${ }^{1}$ David Pisetsky ${ }^{2}$
}

In two papers, investigators from the Karolinska Institutet provide an exciting new perspective on the role of antibodies to citrullinated proteins (anticitrullinated protein antibodies or ACPA) in the pathogenesis of rheumatoid arthritis (RA). ${ }^{1}{ }^{2}$ The data are intriguing and they point to unexpected mechanisms by which these autoantibodies may drive disease manifestations. By revealing a potential role of ACPA in the bone loss and pain that are cardinal features of RA, the investigative teams have advanced a new concept that may (same comment) account for the signs and symptoms of RA at the onset of synovitis as well as during the period of the most intense activity.

The first study by Krishnamurthy et $a l^{1}$ addressed the basis of RA bone loss. Building on prior research on effects of ACPA on bone, ${ }^{3}$ the study demonstrates that polyclonal ACPA derived from patients with RA as well as select monoclonal ACPA can induce osteoclastogensis in human monocytes in vitro. This process appears dependent on a combination of intracellular protein citrullination in the osteoclast as well as autocrine production of (interleukin 8) IL-8. Furthermore, in an animal model, the investigators show that intravenous administration of monoclonal ACPA can induce trabecular bone loss as measured by micro computed tomography (microCT). In this model, the administration of ACPA did not induce synovial inflammation, with the inhibition of bone loss by IL- 8 blockade but not by tumour necrosis factor (TNF) blockade suggesting a mechanism for bone loss beyond inflammation. ${ }^{1}$

The second study by Wigerblad et $a l^{2}$ used similar reagents to explore the effects of ACPA on pain. As the results of

\footnotetext{
${ }^{1}$ VA Palo Alto Health Care System and the Division of Immunology and Rheumatology, Stanford University School of Medicine, Palo Alto, California, USA; ${ }^{2}$ Department of Medicine, Division of Rheumatology, Durham VA Medical Center and Duke University Medical Center, Durham, North Carolina, USA
}

Correspondence to Dr Jeremy Sokolove, VA Palo Alto Health Care System and the Division of Immunology and Rheumatology, Stanford University School of Medicine, Mail Stop 111G, 3801 Miranda Avenue, Palo Alto, CA 94304, USA; sokolove@stanford.edu the study show, the administration of either polyclonal human ACPA or murinised monoclonal ACPA derived from human RA synovium can induce both spontaneous and evoked pain behaviour in mice. This pain began within $24 \mathrm{~h}$ of antibody administration and could persist as long as 28 days in the absence of joint inflammation by histology. Since in vitro incubation of primary neuronal cultures with purified ACPA did not cause significant activation of peripheral neurones, the investigators evaluated whether mechanisms similar to those underlying osteoclastogenesis may also contribute to ACPA-induced hyperalgesia. Extending studies on the effects of chemokine (C-X$\mathrm{C}$ motif) ligand (CXCL) chemokines of nociceptive neurones, ${ }^{3}{ }^{4}$ Wigerblad et $a l^{2}$ showed induction of pain in the ipsilateral paw lasting several hours after intra-articular injection of IL-8. These studies further demonstrated that the administration of CXCL1/2 blocker reparixin can abrogate ACPA-induced pain behaviour (figure 1).

By positing a role of ACPA in bone loss and pain in RA, these studies open a new chapter in a story that began 50 years ago with antiperinuclear factor ${ }^{5}$, continued with antikeratin ${ }^{6}$ and antifilaggrin ${ }^{7}$ anti- $^{8}$ bodies and finally with the work of Schellekens et $a l^{9}$ and Girbal-Neuhauser et $a l^{10}$ who showed that those antibodies present in the sera from patients with RA can recognise proteins containing citrulline, a non-standard amino acid derived from the enzymatic modification of arginine. This work led to development of the highly specific RA biomarker known as the anticyclic citrullinated peptide assay (anti-CCP). As its antigen, this assay uses a citrulline-containing peptide(s). Although, ACPA can bind this peptide, the antigen itself is synthetic and is therefore not present in vivo. Similarly, although ACPA can bind to citrullinated filaggrin or keratin, these epithelial proteins are not expressed in synovial joint suggesting that, like CCP, they may only represent mimics of the true citrullinated targets. Subsequent studies searching the synovial space for ACPA targets have identified many citrullinated molecules including fibrinogen, vimentin and enolase among others although the relevant in vivo target(s) of ACPA is still unknown.

Despite the strong association of APCA with RA, the contribution of these antibodies to pathogenesis nevertheless lacks unequivocal proof. In fact, the expression of ACPA in the blood of patients many years before the clinical onset of RA has called into question a direct role of ACPA as disease mediators rather than serological markers. Although the evolution of the ACPA immune response in the years preceding RA diagnosis predict RA onset, such an association does not prove causality. In this regard, ACPA expression has been associated with increased disease severity, potential for joint erosion ${ }^{11}$ and the occurrence of extra-articular manifestations including cardiovascular ${ }^{12} 13$ and pulmonary diseases. ${ }^{14} 15$ While these findings provide strong, indeed, almost compelling, evidence for a causal link between ACPA and RA manifestations, the evidence remains somewhat circumstantial.

In light of the current two papers in $\mathrm{ARD},{ }^{12}$ it is important to consider previous evidence that ACPA can promote RA pathogenesis. As with any antibody to a self-antigen, ACPA can form immune complexes that can induce inflammation. Work by Clavel et $a l^{16}$ has shown that immune complexes containing citrullinated fibrinogen can activate macrophage cytokine production via a mechanism involving the FcyIIa receptor. Other studies have demonstrated an intrinsic immune capacity of citrullinated proteins and the ability of immune complexes containing citrullinated fibrinogen or histones to drive macrophage cytokine production via costimulation of TLR4 and FcyIIa. ${ }^{17}{ }^{18}$ Furthermore, in vitro experiments have shown that purified ACPA can activate monocytes by binding to a citrullinated GRP78 cell-surface receptor ${ }^{19}$ and driving NF- $\kappa \mathrm{B}$ activation and cytokine production.

Confronting a mix of in vitro and in vivo findings, what is best way to show that an autoantibody is pathogenic? Koch's postulates for an aetiological role of an organism in infection provide a useful framework for thinking about this issue in autoimmunity. In his first postulate, Koch suggested that the 'organism' must be abundant in disease (but not health); the second postulate states that organism should be isolated and cultured from the affected tissue. Applying this standard to ACPA, these antibodies are clearly abundant in both blood and synovial tissue; although antibodies cannot 


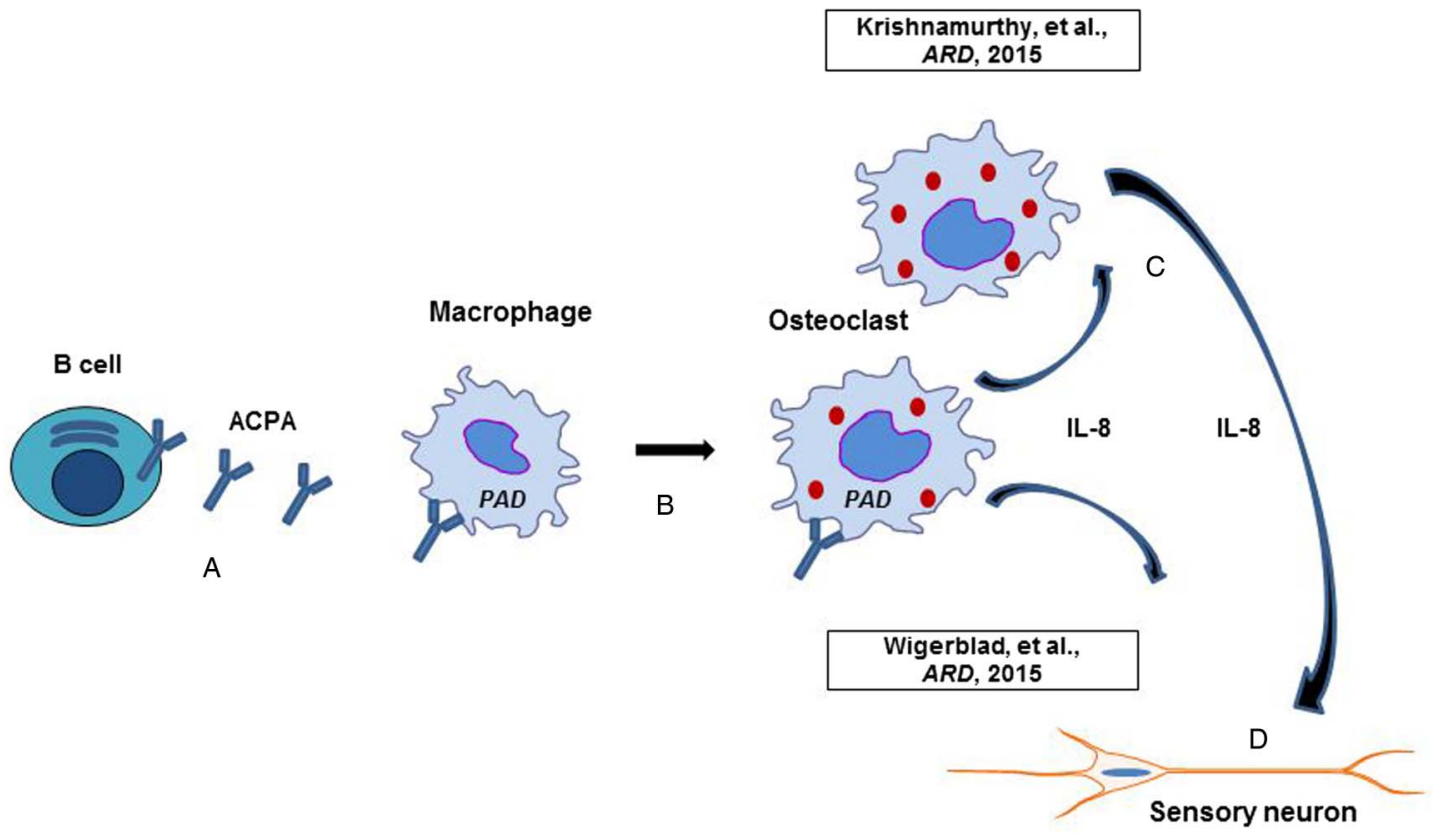

Figure 1 ACPA-induced osteoclastogenesis results in IL-8-dependent induction of bone loss and nociceptive pain. (A) Production of ACPA from activated B cells (B) enhances osteoclast differentiation through at PAD-dependent and (C) IL-8-mediated autocrine loop. (D) Osteoclast-derived IL-8 can additionally induce nociceptive pain via the receptor CXCR1/2. ACPA, anticitrullinated protein antibodies; IL, interleukin; PAD, peptidylarginine deminase.

be 'cultured', several studies have demonstrated ACPA production by synovial and peripheral $B$ cells of patients with RA. ${ }^{20}{ }^{21}$ Koch's third postulate states that the 'organism' should cause disease when introduced into a healthy organism, and according to the fourth postulate, must be reisolated from the inoculated host. Fulfilment of these postulates for ACPA derives from experiments demonstrating that immunisation of animals with a citrullinated antigen can generate ACPA that can induce and transfer disease in murine arthritis models. ${ }^{18} 22-24$

Adapting Koch's now-classic ideas to autoimmunity, Witebsky et $a l^{25}$ formulated analogous postulates, sometimes called Witebsky's postulates. Fulfilment of these postulates requires the ${ }^{1}$ direct demonstration of free, circulating antibodies, ${ }^{2}$ recognition of the specific antigen against which the antibody is directed, ${ }^{26}$ production of antibodies against the same antigen in experimental animals and ${ }^{3}$ appearance of pathological changes similar to those of human disease in the immunised animals. The presence of ACPA in RA is clear. Whether the array of citrullinated proteins bound by ACPA truly constitute a 'known' antigen, however, can be debated. Indeed, the antigens mediating the effects described in the studies of Krishnamurthy et al and Wigerblad et al have not been definitively identified. In vivo studies demonstrating induction of inflammatory arthritis by
ACPA suggest a phenotype analogous to the synovitis observed in RA; however, whether the bone loss and pain in the current studies represent an 'analogous' response to the RA phenotypes is less clear.

Thus, while the two studies are remarkable in suggesting activities of ACPA independent of inflammation, ${ }^{1} 2$ important issues need resolution. Although the polyclonal and monoclonal antibody preparations used in these studies have specificity for a citrullinated antigen, the biochemical identity of the antigen bound in vitro or in vivo in these studies awaits discovery. The study by Krishnamurthy et al ${ }^{1}$ strongly suggests that citrullination within osteoclasts is critical for osteoclastogenesis, but the data provided do not identify the citrullinated molecules generated or the manner in which antibody binding mediates downstream production of IL-8. In this regard, the phenotype observed in that study of Krishnamurthy et al is diffuse trabecular bone loss, not joint erosion. Although RA has been associated with a juxta-articular osteopenia and an increased risk of systemic osteoporosis, ${ }^{27}$ the relationship of these bone phenotypes to RA-associated joint destruction requires further elucidation.

Given the prominence of pain in RA, the experiments by Wigerblad et $a l^{2}$ are provocative, but raise similar questions concerning the mechanisms driving IL-8 production and subsequent changes in nociception. The time course of these responses is notable since the direct intra-articular injection of IL-8 led to only $24 \mathrm{~h}$ of pain behaviour whereas the effects of ACPA administration lasted much longer. How can a single injection of ACPA mediate pain behaviour for 28 days? The circulating half-life of IgG in murine models (even a murinised monoclonal antibody) is likely only 6-8 days. ${ }^{28}$ Thus, the mechanisms leading to hypersensitivity in the absence of inflammation and persistent ACPA exposure are not clear but clearly of great interest.

While the in vivo studies on the role of ACPA in pain and bone loss are impressive, the findings must nevertheless be reconciled with two sets of observations on the relationship of serological and clinical features in RA: the presence of ACPA expression for up to a decade before the onset of RA symptoms and that the ability of disease modifying antirheumatic drug (DMARD) agents to abrogate pain and bone loss without an apparent reduction in levels of ACPA. Although the authors suggest that only some ACPAs are pathogenic, the discordancy in symptoms and ACPA remains a puzzle if these antibodies are truly capable of inducing inflammation-independent pain and/or bone loss.

As the case of all novel research, these papers lead to far more questions than they answer. Nevertheless, they have important implications for thinking about the course of RA and developing new therapy. The fact that seemingly distinct 
pathologic processes (ie, bone loss and pain) may be linked by osteoclast production of IL-8 suggests the value of treatments targeting pathways such as protein citrullination and osteoclast activation as well as neutralisation of IL-8. Further, these studies give impetus for new serological approaches to dissect more finely the specificity of ACPA and to identify more precisely their antigenic targets. In revealing novel faces of ACPA biology, the studies by Krishnamurthy et $a l^{1}$ and Wigerblad $e t a l^{2}$ add new dots to the fascinating picture of the relationship of ACPA and RA pathogenesis. Future studies will build on the far-reaching concepts of these studies and hopefully determine how the dots are really connected.

Contributors All authors contributed to this editorial.

Competing interests None declared.

Provenance and peer review Commissioned; externally peer reviewed.

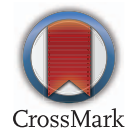

To cite Sokolove J, Pisetsky D. Ann Rheum Dis 2016;75:637-639.

Received 4 November 2015

Revised 17 December 2015

Accepted 20 December 2015

Published Online First 14 January 2016

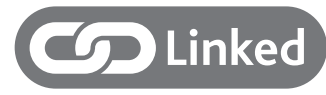

- http://dx.doi.org/10.1136/annrheumdis-2015208094

http://dx.doi.org/10.1136/annrheumdis-2015208093

Ann Rheum Dis 2016;75:637-639.

doi:10.1136/annrheumdis-2015-208308

\section{REFERENCES}

1 Krishnamurthy A, Joshua V, Haj Hensvold A, et al. Identification of a novel chemokine-dependent molecular mechanism underlying rheumatoid arthritis-associated autoantibody-mediated bone loss. Ann Rheum Dis 2016:75:721-9.
2 Wigerblad G, Bas DB, Fernades-Cerqueira C, et al. Autoantibodies to citrullinated proteins induce joint pain independent of inflammation via a chemokine-dependent mechanism. Ann Rheum Dis 2016;75:730-8

3 Qin X, Wan Y, Wang X. CCL2 and CXCL1 trigger calcitonin gene-related peptide release by exciting primary nociceptive neurons. J Neurosci Res 2005;82:51-62.

4 Wang JG, Strong JA, Xie W, et al. The chemokine CXCL1/growth related oncogene increases sodium currents and neuronal excitability in small diameter sensory neurons. Mol Pain 2008;4:38.

5 Nienhuis RLF, Mandema E. A New Serum Factor in Patients with Rheumatoid Arthritis; the Antiperinuclear Factor. Ann Rheum Dis 1964;23:302-5.

6 Young BJ, Mallya RK, Leslie RD, et al. Anti-keratin antibodies in rheumatoid arthritis. $\mathrm{Br}$ Med J 1979;2:97-9.

7 Simon M, Girbal E, Sebbag M, et al. The cytokeratin filament-aggregating protein filaggrin is the target of the so-called "antikeratin antibodies," autoantibodies specific for rheumatoid arthritis. J Clin Invest 1993;92:1387-93.

8 Sebbag $M$, Simon $M$, Vincent $C$, et al. The antiperinuclear factor and the so-called antikeratin antibodies are the same rheumatoid arthritis-specific autoantibodies. J Clin Invest 1995;95:2672-9.

9 Schellekens GA, de Jong BA, van den Hoogen FH, et al. Citrulline is an essential constituent of antigenic determinants recognized by rheumatoid arthritis-specific autoantibodies. J Clin Invest 1998;101:273-81.

10 Girbal-Neuhauser E, Durieux JJ, Arnaud M, et al. The epitopes targeted by the rheumatoid arthritis-associated antifilaggrin autoantibodies are posttranslationally generated on various sites of (pro) filaggrin by deimination of arginine residues. J Immunol 1999;162:585-94.

11 Syversen SW, Gaarder PI, Goll GL, et al. High anti-cyclic citrullinated peptide levels and an algorithm of four variables predict radiographic progression in patients with rheumatoid arthritis: results from a 10 -year longitudinal study. Ann Rheum Dis 2008;67:212-17.

12 Liang KP, Gabriel SE. Autoantibodies: innocent bystander or key player in immunosenescence and atherosclerosis? J Rheumatol 2007;34:1203-7.

13 Goodson NJ, Wiles NJ, Lunt M, et al. Mortality in early inflammatory polyarthritis: cardiovascular mortality is increased in seropositive patients. Arthritis Rheum 2002;46:2010-19.

14 Zhu J, Zhou Y, Chen $X$, et al. A metaanalysis of the increased risk of rheumatoid arthritis-related pulmonary disease as a result of serum anticitrullinated protein antibody positivity. J Rheumatol 2014:41:1282-9.
15 Humphreys JH, van Nies JA, Chipping J, et al. Rheumatoid factor and anti-citrullinated protein antibody positivity, but not level, are associated with increased mortality in patients with rheumatoid arthritis: results from two large independent cohorts. Arthritis Res Ther 2014;16:483.

16 Clavel C, Nogueira L, Laurent L, et al. Induction of macrophage secretion of tumor necrosis factor alpha through Fcgamma receptor lla engagement by rheumatoid arthritis-specific autoantibodies to citrullinated proteins complexed with fibrinogen. Arthritis Rheum 2008;58:678-88.

17 Sokolove J, Zhao X, Chandra PE, et al. Immune complexes containing citrullinated fibrinogen costimulate macrophages via Toll-like receptor 4 and Fcy receptor. Arthritis Rheum 2011;63:53-62.

18 Hyun Sohn D, Rhodes C, Onuma K, et al. Local joint inflammation and histone citrullination provides a murine model for the transition from preclinical autoimmunity to inflammatory arthritis. Arthritis Rheum 2015;67:2877-87.

19 Lu MC, Lai NS, Yu HC, et al. Anti-citrullinated protein antibodies bind surface-expressed citrullinated Grp78 on monocyte/macrophages and stimulate tumor necrosis factor alpha production. Arthritis Rheum 2010;62:1213-23.

20 Amara K, Steen J, Murray F, et al. Monoclonal IgG antibodies generated from joint-derived $B$ cells of RA patients have a strong bias toward citrullinated autoantigen recognition. J Exp Med 2013;210:445-55

21 Tan YC, Kongpachith S, Blum LK, et al. Barcode-enabled sequencing of plasmablast antibody repertoires in rheumatoid arthritis. Arthritis Rheum 2014;66:2706-15.

22 Hill JA, Bell DA, Brintnell W, et al. Arthritis induced by posttranslationally modified (citrullinated) fibrinogen in DR4-IE transgenic mice. J Exp Med 2008:205:967-79.

23 Ho PP, Lee LY, Zhao $X$, et al. Autoimmunity against fibrinogen mediates inflammatory arthritis in mice. J Immunol 2010;184:379-90.

24 Kuhn KA, Kulik L, Tomooka B, et al. Antibodies against citrullinated proteins enhance tissue injury in experimental autoimmune arthritis. J Clin Invest 2006;116:961-73.

25 Witebsky E, Rose NR, Terplan K, et al. Chronic thyroiditis and autoimmunization. I Am Med Assoc 1957;164:1439-47.

26 Harre U, Georgess D, Bang $H$, et al. Induction of osteoclastogenesis and bone loss by human autoantibodies against citrullinated vimentin. J Clin Invest 2012;122:1791-802.

27 Haugeberg G, Ørstavik RE, Kvien TK. Effects of rheumatoid arthritis on bone. Curr Opin Rheumatol 2003; 15:469-75.

28 Vieira P, Rajewsky K. The half-lives of serum immunoglobulins in adult mice. Eur J Immunol 1988;18:313-16. 